Received: $\quad 2018.03 .02$

Accepted: 2018.04.17

Published: 2018.09 .01

\title{
Anticancer Activity of Metformin, an Antidiabetic Drug, Against Ovarian Cancer Cells Involves Inhibition of Cysteine-Rich 61 (Cyr61)/ Akt/Mammalian Target of Rapamycin (mTOR) Signaling Pathway
}

Authors' Contribution: Study Design A Data Collection B Statistical Analysis C Data Interpretation D Manuscript Preparation E Literature Search F Funds Collection G

\author{
BCDEF 1,2 Fengli Zhang* \\ BCDEF 2 Huixiao Chen* \\ BCF 2 Jing Du \\ BD 2 Bin Wang \\ ACDEFG 2 Lixiao Yang
}

1 Infertility Center, Qilu Hospital, Shandong University, Jinan, Shandong, P.R. China 2 Department of Obstetrics and Gynecology, Liaocheng People's Hospital, Liaocheng, Shandong, P.R. China
Corresponding Author: Source of support:
* These authors contribute to this work equally Lixiao Yang, e-mail: YolandeMthu@yahoo.com Departmental sources
Background:

Material/Methods:

Results:

Conclusions:

MeSH Keywords:

Full-text PDF:
Ovarian cancer is considered one of the lethal cancers responsible for high mortality and morbidity across the world. The prognosis and the survival rate of ovarian cancer is far from decent. Cysteine-rich 61 (Cyr61) also known as CCN1, is a member of CCN-family of growth factors, reported to be significantly overexpressed in several malignancies which include, but are not limited to, ovarian cancer. Recent studies have revealed that women with type 2 diabetes mellitus have an elevated risk of ovarian cancer. Hence, administration of an antidiabetic drug with anticancer effects such as metformin may act as an effective therapeutic regime against ovarian cancer.

Cell viability and apoptosis were examined by MTT and Annexin V/PI double staining respectively. Cell migration was determined by Boyden Chamber assay. Transient knockdown of Cyr61 in ovarian cancer cells was achieved by transecting the cells with siRNA for Cyr61using Lipofectamine 2000.

Our results indicated that treatment of ovarian cancer cells with metformin caused significant downregulation of Cyr61 protein expression levels ultimately favoring apoptosis. Transient knockdown of Cyr61 resulted in the inhibition of cell proliferation and migration. This was also associated with the concomitant downregulation of pAkt and pmTOR confirming the role of Cyr61 as an upstream modulator of Akt signaling. Conversely the extracellular supplementation of recombinant Cyr61 attenuates the cytotoxic properties of metformin in ovarian cancer cells.

Taken together, we concluded that metformin exhibits anticancer effects and Cyr61 acts as a direct target for metformin in ovarian cancer cells.

Antineoplastic Agents • Cell Migration Inhibition • Metformin

https://www.medscimonit.com/abstract/index/idArt/909745

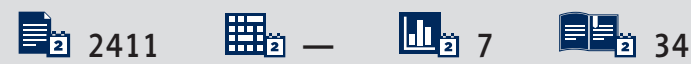




\section{Background}

Ovarian cancer is known to be one of the most lethal gynecological cancers worldwide. It is the seventh most frequently detected cancer and the fifth leading cause of death in the female population around the globe $[1,2]$. The incidence of the ovarian cancer is very high with more than 225000 women being diagnosed for this type of cancer each year [3,4]. Despite several therapeutic strategies, such as radiation and chemotherapy (gemcitabine or cisplatin), the overall 5-year survival rate is less than $40 \%$ [5]. Several epidemiological studies revealed that diabetes increases the risk of several cancers including colorectal, endometrial, breast, and ovarian cancer to name a few. Moreover, diabetes is also associated with poor prognosis and survival of patients suffering from these cancers $[6,7]$. There are a few reports that provide strong evidences about the positive association of diabetes with the development of ovarian cancer [8,9]. The association between diabetes and cancer is complex. From a molecular standpoint, data suggests that elevated insulin-like growth factor I, increased cytokine and estrogen levels, adipokine imbalances, and hyperinsulinemia likely contribute to both an increased risk of ovarian malignancy as well as leading to inferior cancer outcomes [10]. Hence, application of antidiabetic drugs with anticancer activity might prove effective in the treatment of ovarian cancer. Metformin, an oral biguanide medication used to treat type 2 diabetes, has demonstrated potential anticancer properties against several carcinomas and has also been tested in clinical trials against several types of cancers $[11,12]$. Metformin has been evaluated against different types of cancers. For, instance metformin in combination with imatinib was found to effective against colorectal cancer [13]. Similarly, metformin in combination with atorvastatin was found to be effective against prostate cancer [14]. There have been several reports on the effectiveness of metformin on ovarian cancer cells as well [15-17], but the direct target of this drug in ovarian cancer is yet to be reported.

Cysteine-rich 61 (Cyr61), also known as CCN 1 belongs to CCN family (Cyr61/CTGF/Nov) of growth factors and has been reported to be highly expressed in various carcinomas including breast, pancreatic and gastric cancer [18-22]. Cyr61 was also reported to be significantly expressed in ovarian cancer and responsible for the poor prognosis of the disease [23-25]. Recently, Das et al., proposed that metformin inhibits the invasiveness of pancreatic cancer cells by targeting CCN1 signaling [26]. But there is no such report in ovarian cancer. Moreover, it has been demonstrated that CCN 1 modulates $\mathrm{PI3K} / \mathrm{Akt} / \mathrm{mTOR}$ cascade in ovarian cancer cells [23]. In the present study we investigated whether metformin can inhibit the oncogenic potential of ovarian cancer cells by targeting CCN 1 signaling.

\section{Material and Methods}

\section{Cell culture and maintenance}

Human ovarian carcinoma cells OVCAR-3 were cultured in RPMI 1640 complete media containing $10 \%$ fetal bovine serum (FBS) and antibiotics such as penicillin $(100 \mathrm{U} / \mathrm{mL})$ and streptomycin $(100 \mu \mathrm{g} / \mathrm{mL})$ at $37^{\circ} \mathrm{C}$ in a humidified chamber.

\section{Cell viability assay}

Cell viability was assessed spectrophotometrically by using MTT assay. Cultured OVCAR- 3 cells were seeded at the density of $1.2 \times 10^{4}$ to $1.5 \times 10^{4}$ in 96 -well microtiter plates and treated with different concentrations of metformin (Sigma-Aldrich, USA) for 48 hours. Post-treatment, to each well MTT solution was added and finally the ELISA plate reader was used to take the absorbance at $570 \mathrm{~nm}$.

\section{Apoptosis assay}

Apoptosis was determined by Annexin V-FITC/PI dual staining method using BD FACSCalibur and FACS Diva software for analysis. Cultured OVCAR-3 cells at the density of $0.6 \times 10^{6}$ cells/well were seeded in 6-well plates and treated with different concentrations of metformin $(0-20 \mathrm{mM})$ for 48 hours. The Annexin V-FITC apoptosis detection kit (Cayman Chemicals) was finally used to determine in accordance with the manufacturer's protocol.

\section{Matrigel invasion assay}

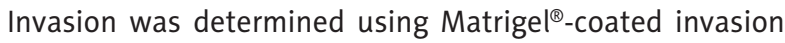
chambers (BD Biosciences). Untreated or metformin (0-20 mM) treated cells, reached the lower surface of the membrane were stained with crystal violet (CV). Images of CV-stained cells were captured by a phase contrast microscope. Acetic acid (10\%) was used to dissolve the resulting CV complex. Finally, the cell invasion was determined by taking the absorbance at $600 \mathrm{~nm}$.

\section{Transient transfection with siCyr61}

For transient knockdown of Cyr61 in OVCAR-3 cells, siCyr61 RNA (sc-39331) and scrambled siRNAs (sc-44234) were purchased from Santa Cruz and cells were transfected with $100 \mathrm{nM}$ of siCyr61 RNA using Lipofectamine 2000 (Invitrogen), following the manufacturer's protocol.

\section{Western blot analysis}

The cultured OVCAR-3 cells were harvested and lysed with lysis buffer. The protein extracts were incubated at $99^{\circ} \mathrm{C}$ for $15 \mathrm{~min}$ in the presence of loading buffer followed by separation of cell 
extracts using 15\% SDS-PAGE gel. The samples were then put onto polyvinylidene fluoride membranes blocked using $5 \%$ skimmed milk powder. Membrane incubation with primary antibodies (Cry61, Bcl-2, Bax, cleaved caspase-3, AKT, PI3K, mTOR, p-AKT, p-PI3K, and p-mTOR) was performed for 24 hours at $4^{\circ} \mathrm{C}$. The membranes were incubated with horseradish peroxidaselinked secondary biotinylated secondary antibodies at 1: 1000 dilution for 2 hours. Washing of the membranes with PBS was followed by visualization of the immune-reactive bands with the help of ECL-PLUS kit according to the guidelines of the manufacturer. The immune complexes development was carried out using an ECL detection kit according to the manual. The bands were analyzed using GelGDoc2000 imaging system.

\section{Statistical analysis}

All data were expressed as the mean \pm standard deviation (SD). Statistically significant differences between groups were determined by the students $t$-test for comparisons between 2 samples and the one-way ANNOVA followed by Tukey's post hoc test for comparisons between more than 2 samples, and by using GraphPad Prism 7 software (GraphPad Software, Inc., La Jolla, CA, USA). A value of $P<0.05$ was considered statistically significant.

\section{Results}

\section{Metformin reduced viability of ovarian cancer cells and induced apoptosis}

Treatment of human ovarian cancer cells OVCAR-3 with metformin resulted in the significant loss of cell viability in a dosedependent manner (Figure 1A). Treatment of OVCAR-3 with different concentrations of metformin for 48 hours caused a gradual decrease in cell viability with an $\mathrm{IC}_{50}$ of $30 \mathrm{mM}$.

To determine the mode of cell death, we investigated if metformin could induce apoptosis in metformin-treated OVCAR-3 cells. The results showed a gradual increase in apoptotic population in metformin-treated cells with increased concentration of metformin (Figure 1B). In the presence of $20 \mathrm{mM}$ metformin, the apoptotic cell populations increased up to $11.3 \%$, while at $I C_{50}$ dose it exhibited about $29.6 \%$ increase. Moreover, the expression of the pro-apoptotic markers Bax and cl-caspase- 3 showed significant upregulation with a concomitant decrease in the expression of the anti-apoptotic protein $\mathrm{Bcl}-2$ (Figure 1C).

\section{Metformin inhibits colony forming ability of ovarian cancer cells}

The clonogenic potential of the ovarian cancer cells was found to be significantly reduced in the presence of metformin (Figure 2A, 2B). When seeded at low density, OVCAR-3 cells formed viable colonies after 5 days of culture. However, in the presence of metformin, a marked reduction in the number of OVCAR-3 colonies was observed. At the concentration of $10 \mathrm{mM}$ metformin, the colony forming potential of OVCAR-3 cells was decreased by $60 \%$ with further decrease with increased concentration of metformin.

\section{Metformin inhibited invasiveness of ovarian cancer cells}

The invasive properties of OVCAR-3 cells were found to be significantly inhibited in the presence of metformin, as determined by Boyden Chamber Matrigel assay (Figure 3A, 3B). The invasive properties of OVCAR-3 cells were reduced by $50 \%$ in the presence of $10 \mathrm{mM}$ metformin, while more inhibition was observed at higher doses of metformin.

\section{Depletion of Cyr61 affected viability and invasiveness in ovarian cancer cells by modulating PI3K/Akt/mTOR signaling}

It has been previously reported that Cyr61 plays an important role in the progression of ovarian cancer [23-25]. Hence, we investigated the effect of Cyr61 knockdown on the tumorigenic properties of ovarian cancer cells. SiRNA mediated knockdown of Cyr61 resulted in the loss of viability and inhibition of invasive properties of OVCAR-3 cells (Figure 4A-4D). Furthermore, the expression of some of the important proteins of PI3K/Akt/ mTOR signaling pathway was also found to be downregulated in Cyr61 ${ }^{\text {-ve }}$ cells, which corroborates with the findings reported in earlier studies [23] (Figure 4E).

\section{Metformin targeted PI3K/Akt/mTOR signaling in ovarian cancer cells}

To investigate the impact of metformin on PI3K/Akt/mTOR signaling, we determined the phosphorylation levels of $p 85$, the regulatory subunit of $\mathrm{PI} 3 \mathrm{~K}, \mathrm{Akt}$, and mTOR in metformintreated OVCAR-3 cells. Interestingly, it was observed that metformin administration caused a significant reduction of $\mathrm{p} 85$, pAkt (Ser 473), and p-mTOR (Ser2448) levels in OVCAR-3 cells (Figure 5A-5D). However, the total Akt and mTOR expression levels remained unaltered.

\section{Cyr61 acted as a direct target of metformin in ovarian cancer cells}

To investigate whether Cyr61 acts as a target for metformin, we determined the expression levels of Cyr61 in metformintreated OVCAR-3 cells. We observed a dose-dependent decrease in Cyr61 expression in metformin-treated OVCAR-3 cells (Figure 6A, 6B). To further confirm that this decline in Cyr61 expression by metformin affects the viability of OVCAR-3, we co-incubated cells with both metformin and different 


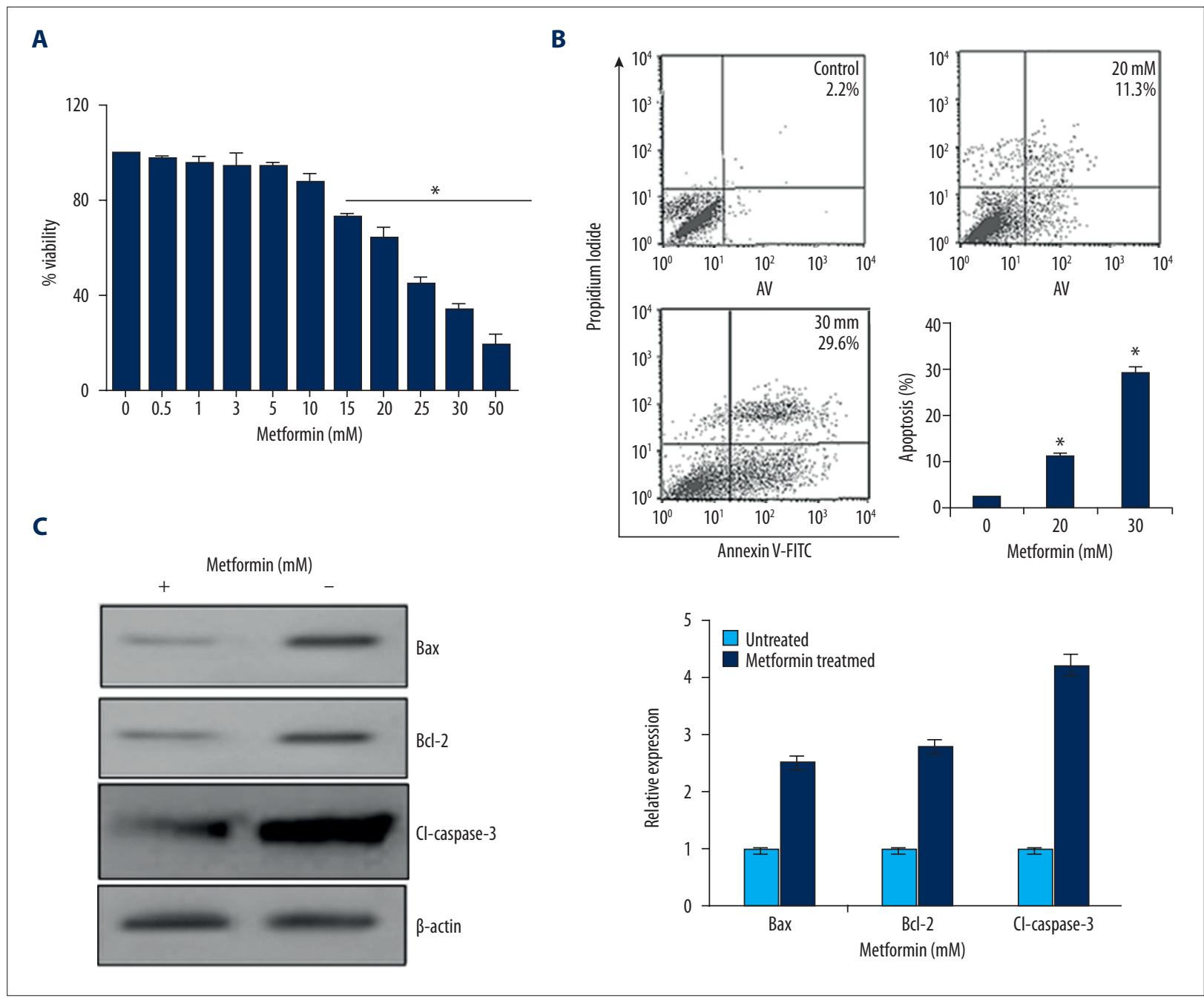

Figure 1. Metformin induces apoptosis in ovarian cancer cells. (A) Cell viability of OVCAR-3 cells in the presence of metformin for 48 hours as determined by MTT assay. (B) Apoptosis assay for OVCAR-3 cells treated with metformin as determined by Annexin V/PI double staining. (C) Modulation of pro- and anti-apoptotic markers. The results are expressed as mean \pm standard deviation of 3 independent experiments. The values were considered significant at ${ }^{*} P<0.05$.

concentrations of human recombinant Cyr61. It was observed that the viability of metformin-treated OVCAR-3 cells was restored in the presence of recombinant Cyr61 protein (Figure 6C). Thus, it might be concluded that Cyr61 acted as a direct target for metformin in ovarian cancer cells.

\section{Depletion of Cyr61 sanitized ovarian cancer cells to PTX treatment}

Previous reports suggested that Cyr61 was responsible for the acquisition of paclitaxel (PTX) resistance in various carcinomas $[27,28]$. Hence, we investigated whether Cyr 61 played any role in PTX resistance in ovarian cancer cells. Treatment of OVCAR-3 with PTX resulted in a dose-dependent inhibition of cell viability and the $\mathrm{IC}_{50}$ was observed at around $600 \mathrm{nM}$. But when Cyr61-ve OVCAR-3 cells were treated with PTX, a drastic decrease in cell viability was observed and the $\mathrm{IC}_{50}$ was observed at around $150 \mathrm{nM}$ (Figure 7A). Thus, we conclude that Cyr61 was responsible for the acquisition of PTX resistance in ovarian cancer cells.

\section{Metformin enhanced chemosensitivity of ovarian cells}

Since metformin treatment caused significant decrease in the expression of Cyr61, we further investigated whether it could enhance the efficacy of PTX against ovarian cancer cells. Treatment of OVCAR-3 cells with metformin or PTX alone resulted in the loss of viability by $11 \%$ and $20 \%$ respectively. But when metformin was administered in combination with PTX, a drastic decrease in the cell viability was observed (Figure 7B). The results showed that co-administered of metformin with PTX caused $35 \%$ reduction in the viability of OVCAR-3 cells. Interestingly, 


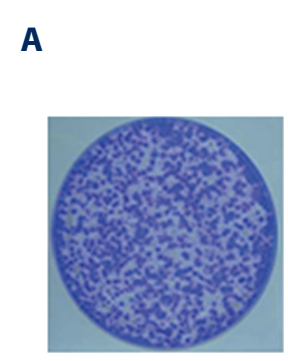

Control

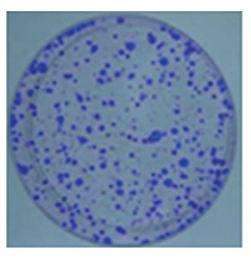

$10 \mathrm{nM}$

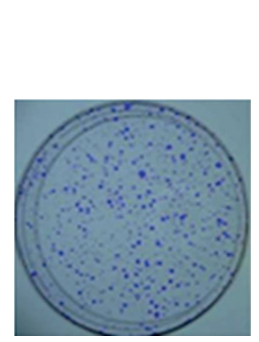

$20 \mathrm{nM}$
B

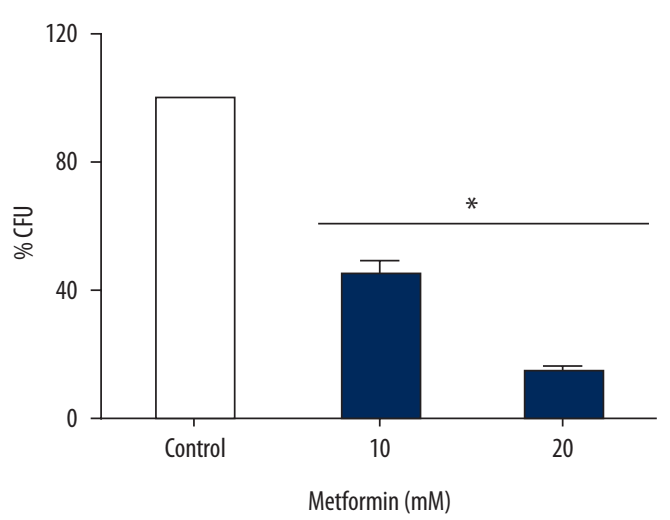

Figure 2. Metformin inhibits colony formation in ovarian cancer cells. (A) Photographs of viable colonies of OVCAR-3 cells in the presence or absence of metformin, stained with crystal violet; (B) \% colony forming unit (CFU), determined spectrophotometrically. The results are expressed as mean \pm standard deviation of 3 independent experiments. The values were considered significant at ${ }^{*} P<0.05$.

A
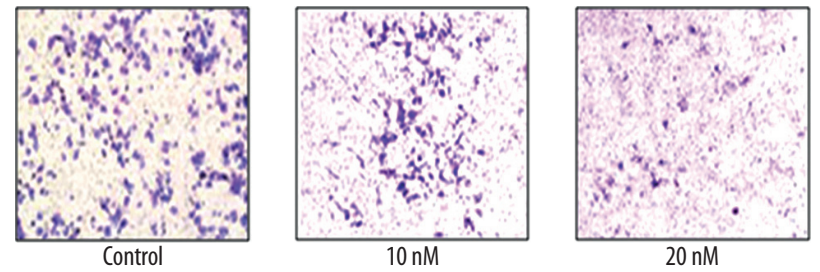

B

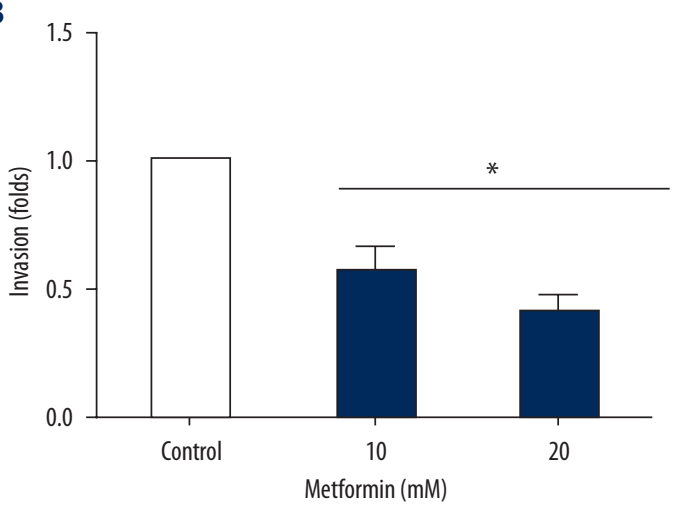

Figure 3. Metformin inhibits invasiveness of ovarian cancer cells. (A) Invasion was determined by the Boyden Chamber assay. (B) Extent of invasion as determined spectrophotometrically. The results are expressed as mean \pm standard deviation of 3 independent experiments. The values were considered significant at ${ }^{*} P<0.05$.

when the cells were pre-incubated with metformin and then treated with PTX, cell viability was decreased by about $65 \%$. Taken together, these results indicate that metformin increases the therapeutic potential of PTX in ovarian cancer cells.

\section{Discussion}

The PI3K/AKT/mTOR cascade plays an important role in the growth, propagation, and metastasis of several human malignancies, including the ovarian cancer [29-31]. Previous genomic studies have suggested that this pathway undergoes more frequent mutational alterations in ovarian carcinoma compared to other malignancies [32]. Accumulating experimental evidence also indicates that PI3K/AKT/mTOR signaling is responsible for the acquisition of chemoresistance properties and poor prognoses of ovarian cancer [33,34]. Cyr61, a member of CCN 1 family of growth factors, has been known to be highly expressed in ovarian cancer cells/tissues and associated with poor prognosis of the disease [23-25]. Furthermore, Cyr61 has also been reported to modulate PI3K/AKT/mTOR signaling in ovarian cancer cells [23]. Although several PI3K/ AKT/mTOR signaling inhibitors have undergone clinical trials, the efficacy rate has not been very optimistic due to frequent mutations, heterogeneity, complexity of the disease, toxicity, and development of chemoresistance phenotype [29]. Hence, development of novel drug candidates has become an urgent requirement for the prevention of ovarian cancer.

Recently, Das et al., reported that the antidiabetic drug metformin inhibits the oncogenic potential of pancreatic cancer cells by targeting Cyr61 signaling [26]. Further literature search revealed 


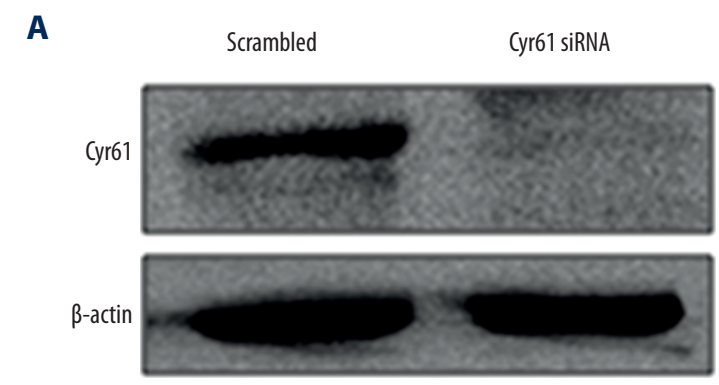

C

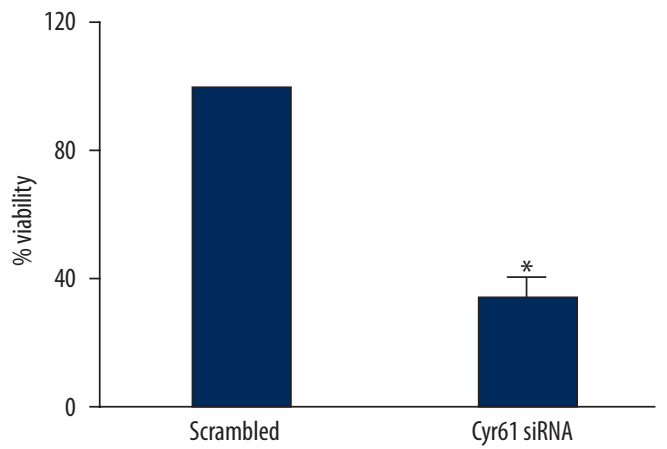

D

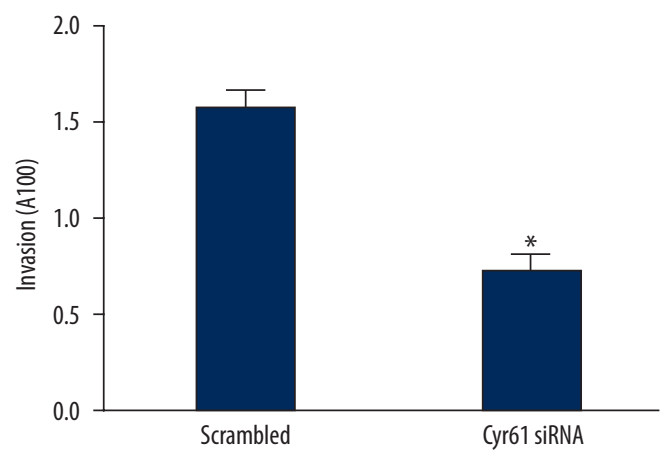

B

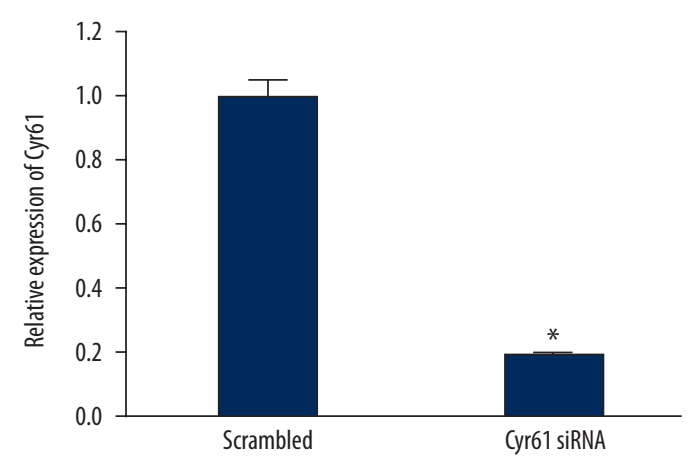

E
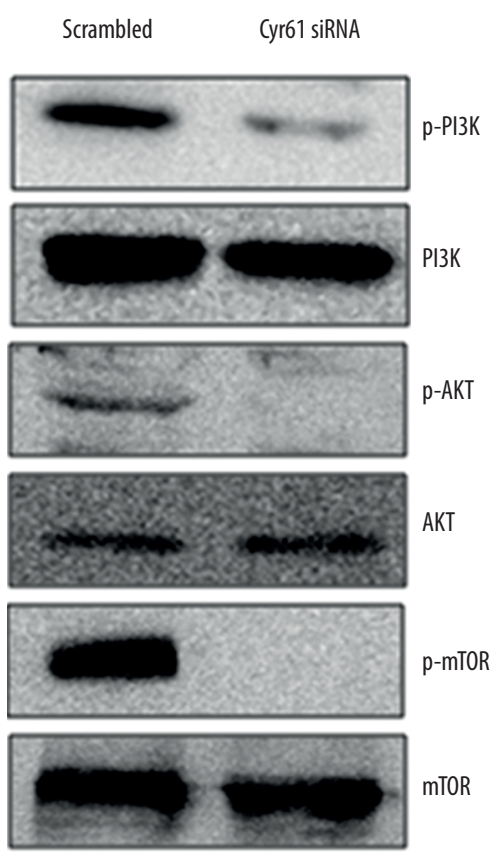

Figure 4. Effect of Cyr61 on oncogenic potential and PI3K/Akt/mTOR axis in ovarian cancer cells. (A) Transient knockdown of Cyr61 by siRNA method in OVCAR-3 cells. (B) qRT-PCR analysis showing the expression of Cyr61 in Cyr61SiRNA transfected OVCAR-3 cells. (C) Viability of Cyr61 siRNA and scrambled siRNA transfected OVCAR-3 cells. (D) Boyden Chamber invasion assay of Cyr61 siRNA and scrambled siRNA transfected OVCAR-3 cells. (E) PI3K/Akt/mTOR axis in OVCAR-3 cells. All the experiments were carried out in triplicates. The values were considered significant at ${ }^{*} P<0.05$.

that female patients suffering from diabetes were more prone to the risk of ovarian cancer [8-10]. Although the effectiveness of metformin on ovarian cancer cells have been reported [15-17], the precise mechanism of this drug in ovarian cancer cells is not yet understood. Hence, in the present study, we thoroughly investigated whether Cyr61, being the upstream modulator of $\mathrm{PI3K} / \mathrm{AKT} / \mathrm{mTOR}$ pathway, acts as a direct target for metformin.

In our study we have observed that metformin treatment resulted in the significant loss of cell viability in ovarian cancer cells OVCAR-3, along with the simultaneous induction of apoptosis (Figure 1). Moreover, metformin also inhibited the colony forming ability and invasiveness of OVCAR-3 cells in a dose-dependent manner (Figures 2, 3). SiRNA mediated knockdown of Cyr61 significantly downregulated the PI3K/AKT/mTOR pathway in OVCAR-3 cells (Figure 4) and similar results were observed when the cells were treated with metformin (Figure 5). Furthermore, it was observed that metformin treatment resulted in dose-dependent decrease of Cyr61 expression (Figure 6). However, when OVCAR-3 cells were co-treated with human recombinant Cyr61, the cytotoxic effects of metformin on ovarian cancer cells were nullified to a significant extent (Figure 6). 
A

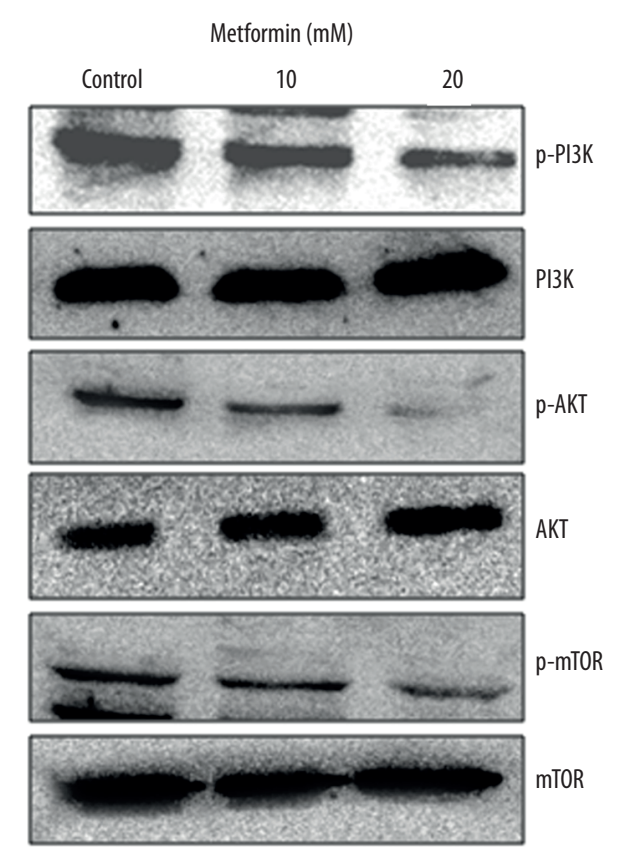

C

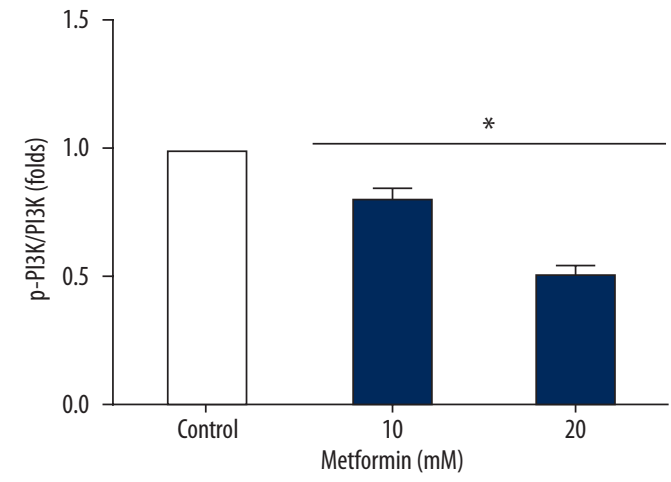

B

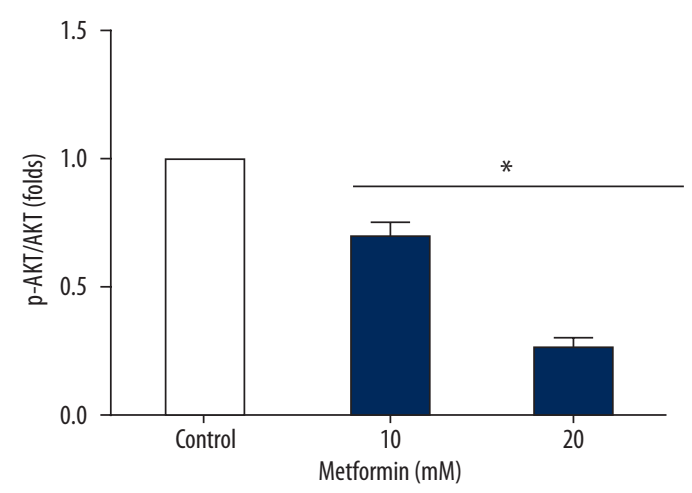

D

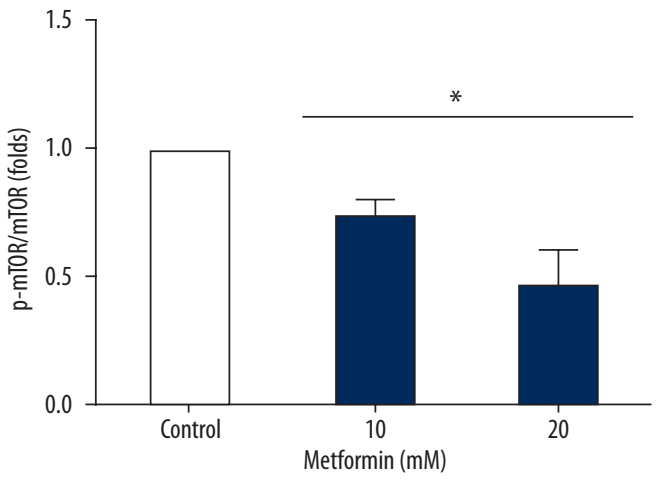

Figure 5. Effect of metformin on the PI3K/Akt/mTOR pathway. Cultured OVCAR-3 cells were treated with different doses of metformin (0-20 mM) for 48 hours and western blots for p-Akt (Ser 473), Akt, p-mTOR (Ser 2448) and mTOR were performed.

(A) Western blot analysis for PI3K (p85), PI3K, p-Akt Ser 473, Akt, p-mTOR Ser 2448, mTOR, and $\beta$-actin used as a loading control. Relative band intensities for (B) p-PI3K (p85), (C) p-Akt ${ }^{\text {Ser }}{ }^{473}$, and (D) p-mTor Ser 2448 . The results are expressed as mean \pm standard deviation of 3 independent experiments. The values were considered significant at ${ }^{*} P<0.05$.

This data confirmed the direct involvement of Cyr61 in metformin-mediated cytotoxicity in ovarian cancer cells. Since the positive association of Cyr61 with drug resistance in several carcinomas has been well documented, we further investigated whether it had similar roles in ovarian cancer. Comparative cytotoxicity studies revealed that in Cyr61-depleted OVCAR-3 cells, the effect of PTX was more prominent than the effect in parental cells (Figure 7A). Since metformin targets Cyr61 in ovarian cancer cells, we presumed that it might enhance the efficacy of PTX on ovarian cancer cells. It was observed that co-treatment of OVCAR-3 cells with both metformin and PTX resulted in significant reduction of cell viability compared to the drugs when administered alone. Also, when the cells that were pretreated with metformin, were further post-incubated with PTX, cell viability was more drastically reduced. Since it is well established that diabetic patients have a very high risk of developing ovarian cancer, metformin can serve the dual purpose of combating both pathological conditions. Although, this study was carried out using only 1 cell model, more studies using many more cell lines and in vivo evaluation of metformin will surely pave the way for development of metformin as an anticancer agent for the treatment of ovarian cancer. 


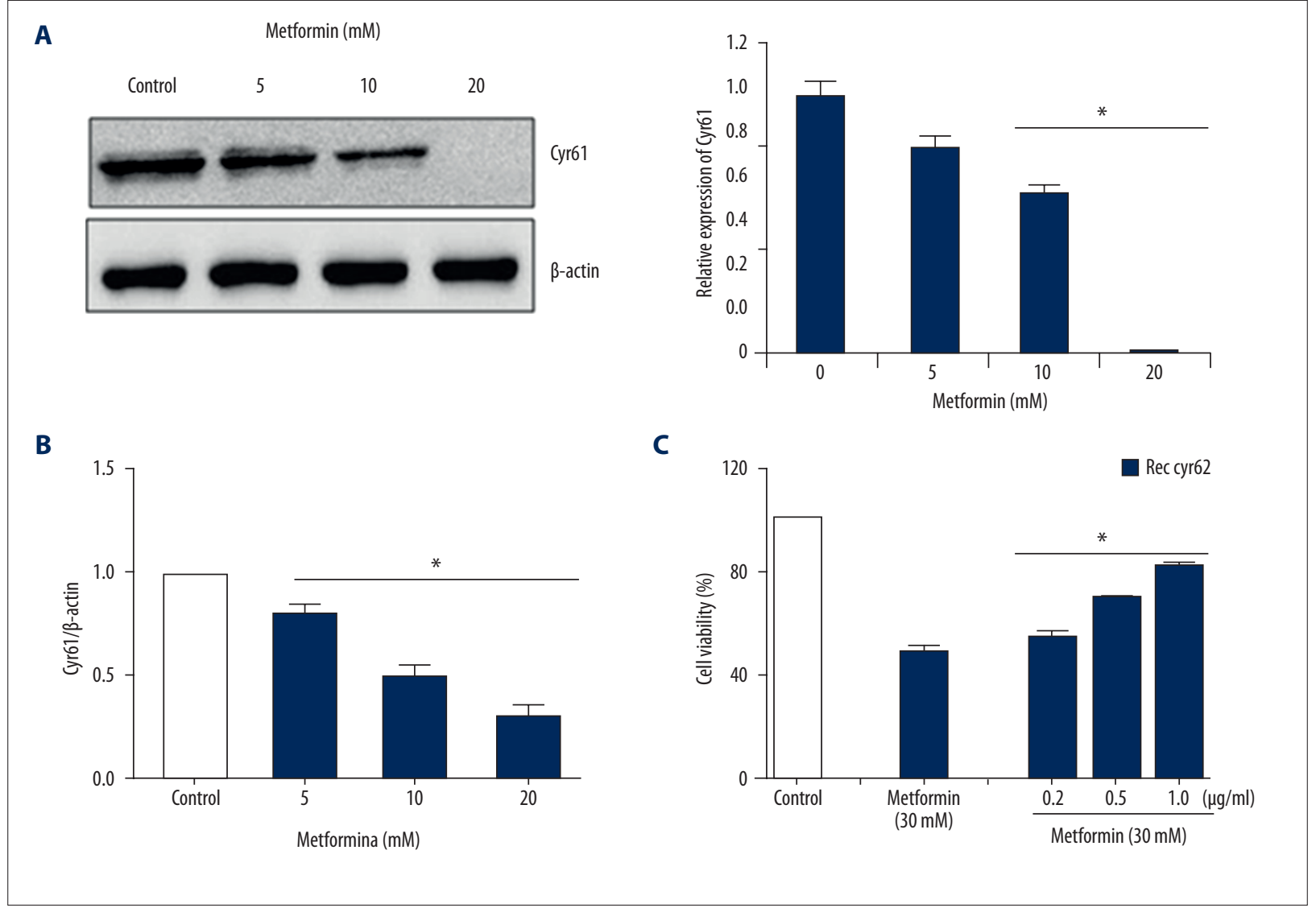

Figure 6. Cyr61 regulates metformin action in ovarian cancer cells (A) and (B) the effect of metformin on Cyr61 expression in OVCAR-3 cells. (C) Viability of metformin-treated OVCAR-3 cells in the presence of human recombinant Cyr61. All the experiments were carried out in triplicates. The values were considered significant at * $P<0.05$.
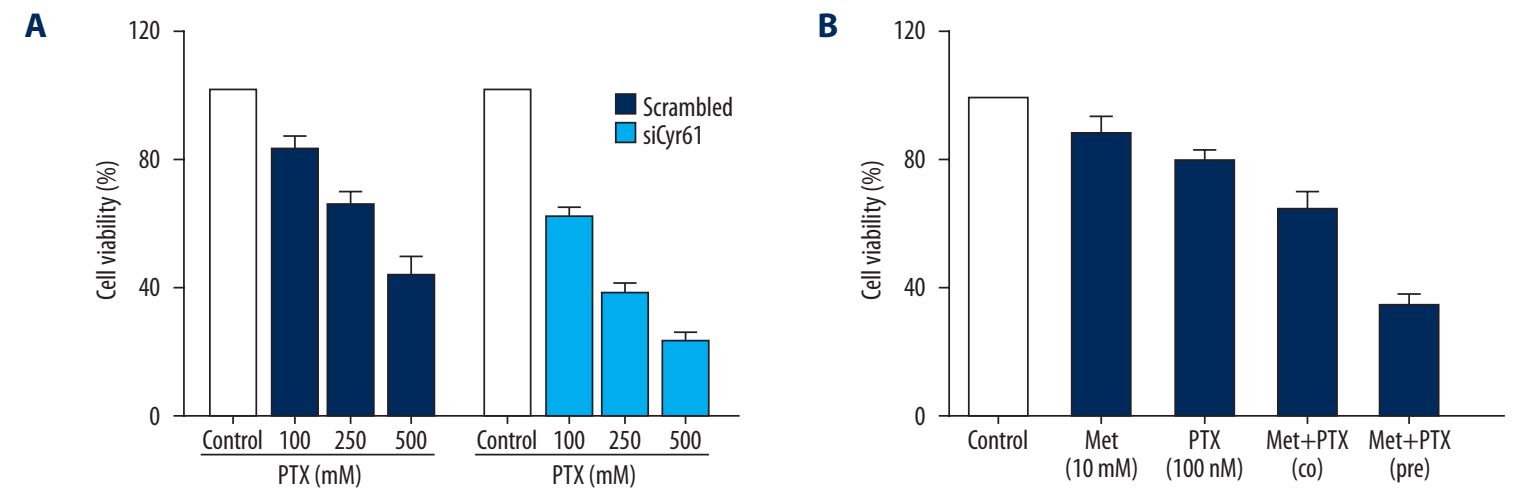

Figure 7. Cyr61 regulates chemoresistance properties of ovarian cancer cells. (A) Cell viability of Cyr61 siRNA and scrambled siRNA transfected OVCAR-3 cells in the presence of paclitaxel. (B) Combination of paclitaxel and metformin.

\section{Conclusions}

These study results suggest that metformin inhibits the oncogenic potential of ovarian cancer cells and enhances chemosensitivity by targeting the Cyr61/PI3K/AKT/mTOR axis. Metformin is already an approved drug for the treatment of diabetes and there are no cytotoxicity issues associated with its use. Therefore, it may prove a potential candidate for the treatment of ovarian cancer. 


\section{References:}

1. Jayson GC, Kohn EC, Kitchener HC, Ledermann JA: Ovarian cancer. Lancet, 2014; 384(9951): 1376-88

2. Nam MS, Jung DB, Seo KH et al: Apoptotic effect of Sanggenol L via caspase activation and inhibition of NF-kappaB signaling in ovarian cancer cells. Phytother Res, 2016; 30(1): 90-96

3. Ivanov S, Ivanov S, Khadzhiolov N: [Ovarian tumours - accuracy of frozen section diagnosis]. Akush Ginekol (Sofiia), 2005; 44(1): 11-13 [in Bulgarian]

4. Saika K, Sobue T: [Cancer statistics in the world]. Gan To Kagaku Ryoho, 2013; 40(13): 2475-80 [in Japanese]

5. Edwards SJ, Barton S, Thurgar E, Trevor N: Topotecan, pegylated liposomal doxorubicin hydrochloride, paclitaxel, trabectedin and gemcitabine for advanced recurrent or refractory ovarian cancer: A systematic review and economic evaluation. Health Technol Assess, 2015; 19(7): 1-480

6. Coughlin SS, Calle EE, Teras LR et al: Diabetes mellitus as a predictor of cancer mortality in a large cohort of US adults. Am J Epidemiol, 2004; 159(12) 1160-67

7. Vigneri $P$, Frasca F, Sciacca L et al: Diabetes and cancer. Endocr Relat Cancer, 2009; 16(4): 1103-23

8. Craig ER, Londono Al, Norian LA, Arend RC: Metabolic risk factors and mechanisms of disease in epithelial ovarian cancer: A review. Gynecol Oncol, 2016; 143(3): 674-83

9. Zhang D, Zhao Y, Wang T et al: Diabetes mellitus and long-term mortality of ovarian cancer patients. A systematic review and meta-analysis of 12 cohort studies. Diabetes Metab Res Rev, 2016; 33; 14-21

10. Shah MM, Erickson BK, Matin T et al: Diabetes mellitus and ovarian cancer: More complex than just increasing risk. Gynecol Oncol, 2014; 135(2): 273-77

11. Chae YK, Arya A, Malecek MK et al: Repurposing metformin for cancer treatment: Current clinical studies. Oncotarget, 2016; 7(26): 40767-80

12. Daugan M, Dufay Wojcicki A et al: Metformin: An anti-diabetic drug to fight cancer. Pharmacol Res, 2016; 113: 675-85

13. Lee J, Park D, Lee Y: Metformin synergistically potentiates the antitumor effects of imatinib in colorectal cancer cells. Dev Reprod, 2017; 21: 139-50

14. Wang ZS, Huang HR, Zhang LY et al: Mechanistic study of inhibitory effects of metformin and atorvastatin in combination on prostate cancer cells in vitro and in vivo. Biol Pharm Bull, 2017; 40: 1247-54

15. Dilokthornsakul $P$, Chaiyakunapruk N, Termrungruanglert $W$ et al: The effects of metformin on ovarian cancer: A systematic review. Int J Gynecol Cancer, 2013; 23(9): 1544-51

16. Gadducci A, Biglia N, Tana R et al: Metformin use and gynecological cancers: A novel treatment option emerging from drug repositioning. Crit Rev Oncol Hematol, 2016; 105: 73-83

17. Li B, Li X, Ni Z et al: Dichloroacetate and metformin synergistically suppress the growth of ovarian cancer cells. Oncotarget, 2016; 7(37): 59458-70

18. Tsai MS, Bogart DF, Castaneda JM et al: Cyr61 promotes breast tumorigenesis and cancer progression. Oncogene, 2002; 21(53): 8178-85

19. Tsai MS, Bogart DF, Li P et al: Expression and regulation of Cyr61 in human breast cancer cell lines. Oncogene, 2002; 21(6): 964-73
20. Haque I, Mehta S, Majumder M et al: Cyr61/CCN1 signaling is critical for epithelial-mesenchymal transition and stemness and promotes pancreatic carcinogenesis. Mol Cancer, 2011; 10: 8

21. Wei J, Yu G, Shao G et al: CYR61 (CCN1) is a metastatic biomarker of gastric cardia adenocarcinoma. Oncotarget, 2016; 7(21): 31067-78

22. Lee H, Hwang SJ, Kim HR et al: Neurofibromatosis 2 (NF2) controls the invasiveness of glioblastoma through YAP-dependent expression of CYR61 CCN1 and miR-296-3p. Biochim Biophys Acta, 2016; 1859(4): 599-611

23. Lee KB, Byun HJ, Park SH et al: CYR61 controls p53 and NF-kappaB expression through $\mathrm{PI} 3 \mathrm{~K} / \mathrm{Akt} / \mathrm{mTOR}$ pathways in carboplatin-induced ovarian cancer cells. Cancer Lett, 2012; 315(1): 86-95

24. Shen H, Cai M, Zhao S et al: CYR61 overexpression associated with the development and poor prognosis of ovarian carcinoma. Med Oncol, 2014; 31(8): 117

25. Gery S, Xie D, Yin D et al: Ovarian carcinomas: CCN genes are aberrantly expressed and CCN1 promotes proliferation of these cells. Clin Cancer Res, 2005; 11(20): 7243-54

26. Das A DA, Haque I, Maity $G$ et al: Metformin inhibits the oncogenic potential and invasiveness of pancreatic cancer cells targeting CCN1-CXCR4 axis: A new perspective for an old antidiabetic drug [Proceedings of the $106^{\text {th }}$ Annual Meeting of the American Association for Cancer Research]. Cancer Res, 2015; 75(15 Suppl.): Abstract 3572

27. Lai $D$, Ho KC, Hao Y, Yang X: Taxol resistance in breast cancer cells is mediated by the hippo pathway component TAZ and its downstream transcriptional targets Cyr61 and CTGF. Cancer Res, 2011; 71(7): 2728-38

28. Menendez JA, Vellon L, Mehmi I et al: A novel CYR61-triggered 'CYR61alphavbeta3 integrin loop' regulates breast cancer cell survival and chemosensitivity through activation of ERK1/ERK2 MAPK signaling pathway. Oncogene, 2005; 24(5): 761-79

29. Cheaib B, Auguste A, Leary A: The PI3K/Akt/mTOR pathway in ovarian cancer: Therapeutic opportunities and challenges. Chin J Cancer, 2015; 34(1): 4-16

30. Mabuchi S, Kuroda H, Takahashi R, Sasano T: The PI3K/AKT/mTOR pathway as a therapeutic target in ovarian cancer. Gynecol Oncol, 2015; 137(1): 173-79

31. Bai H, Li H, Li W et al: The PI3K/AKT/mTOR pathway is a potential predictor of distinct invasive and migratory capacities in human ovarian cancer cell lines. Oncotarget, 2015; 6(28): 25520-32

32. Huang J, Zhang L, Greshock J et al: Frequent genetic abnormalities of the $\mathrm{PI3K} / \mathrm{AKT}$ pathway in primary ovarian cancer predict patient outcome. Genes Chromosomes Cancer, 2011; 50(8): 606-18

33. Carden CP, Stewart A, Thavasu P et al: The association of PI3 kinase signaling and chemoresistance in advanced ovarian cancer. Mol Cancer Ther, 2012; 11(7): 1609-17

34. Kolasa IK, Rembiszewska A, Felisiak A et al: PIK3CA amplification associates with resistance to chemotherapy in ovarian cancer patients. Cance Biol Ther, 2009; 8(1): 21-26 